\title{
Modified Phase-Scheduled-Command FxLMS Algorithm for Active Sound Profiling
}

\author{
Vinal Patel, Jordan Cheer*, and Nithin V. George, Member, IEEE
}

\begin{abstract}
Active sound profiling, or active noise equalization strategies have been proposed to achieve spectral shaping of a primary disturbance signal. The control algorithms proposed to achieve such spectral shaping have either suffered from poor robustness to plant modelling uncertainties or required high levels of control effort. To improve the robustness of active sound profiling to uncertainties in the plant model, whilst avoiding increased control effort, a modified phase-scheduledcommand filtered-x least-mean-square (FxLMS) algorithm is proposed in this paper. The new algorithm provides improved stability, whilst requiring the minimum control effort. This improvement is achieved by replacing the plant model with an intelligent adaptive-hysteresis switching mechanism to allow the necessary estimation of the disturbance signal phase. The improved performance and robustness of the proposed algorithm is demonstrated through a series of simulations using measured acoustic responses.
\end{abstract}

Index Terms-Active sound profiling, phase-scheduledcommand FxLMS, automatic-phase-command FxLMS, active noise equalization, hysteresis switch.

\section{INTRODUCTION}

Active noise control (ANC), which is based on the principle of destructive interference, has been found to be an effective candidate for noise mitigation in the low-frequency region [1]-[6]. In a feed-forward ANC system, a reference sensor is used to measure the noise to be cancelled, a loudspeaker is employed to generate the necessary anti-noise, and an error microphone is used to measure the level of noise cancellation achieved. The signal driving the loudspeaker can be controlled using an adaptive finite-impulse-response (FIR) filter. The weights of the filter are updated using a suitable algorithm, which aims to minimize the residual noise [7]. One of the most widely employed algorithms used for ANC is the filtered- $x$ least-mean-square (FxLMS) algorithm [2].

In some applications, it is desirable to retain a residual error with a specified spectral shape. For example, it may be sensible to modify the spectral shape of the disturbance noise in automobiles [8], [9], aircraft [10], [11], air-conditioning systems [12], [13], or incubators [14] in order to control subjective metrics such as loudness, sharpness, or roughness [15] rather than the sound pressure level. For such applications,

J. Cheer is with the Institute of Sound and Vibration Research (ISVR), University of Southampton, Southampton SO17 1BJ, U.K.(e-mail: j.cheer@soton.ac.uk).

V. Patel and N. V. George are with the Department of Electrical Engineering, Indian Institute of Technology Gandhinagar, India. (e-mail: vinal.patel@iitgn.ac.in; nithin@iitgn.ac.in). The work of V. Patel and N V. George was supported by the Department of Science and Technology, Government of India under the Fast Track Scheme for Young Scientists (SERB/ET-0018/2013) researchers have modified the active control strategy so that the central focus is not only on cancellation, but could also be on either the attenuation or enhancement of a primary disturbance signal. This process is called active sound profiling [16] or active noise equalization [17]. A narrowband active noise equalizer was proposed in [17], in which an FxLMS algorithm was used to update the weights of the controller to obtain residual noise shaping. A similar approach using the filterederror least-mean-square (FeLMS) algorithm to overcome problems with enhancements in the passband was reported in [18]. However, the sound profiling scheme proposed in [17], [18] has been shown to become unstable in the presence of errors in the plant model, especially at high enhancement gains [19]. In order to overcome these limitations, a phasescheduled-command FxLMS (PSC-FXLMS) algorithm and an automatic-phase-command FxLMS (APC-FxLMS) algorithm were presented in [19].

The above mentioned sound profiling schemes use an additional internal model of the plant to obtain an estimate of the disturbance signal, which is used to adapt the sound profiling. The stability of these sound profiling schemes is, therefore, dependent on the accuracy of this plant model. The presence of modelling errors in these algorithms may lead to instability and are also more sensitive to such modelling errors than the standard FxLMS algorithm [19]. In [19] it was shown that PSC-FxLMS becomes unstable due to the presence of phase errors in the plant model during enhancement and APCFxLMS was proposed to overcome this limitation. Although APC-FxLMS is able to remain stable whilst operating in enhancement mode in the presence of errors in the plant model, it requires a larger control effort to achieve the desired level.

In practical active control systems, the physical plant response, characterized by the response from the loudspeaker input to the error microphone output, is susceptible to variations over time. These variations might be caused by changes in the ambient temperature or humidity, fatigue in the control actuators and error sensors, or practical changes in the acoustic environment due to, for example, changes in the occupancy of the acoustic space. Due to the dynamic nature of the physical plant, as well as truncation error introduced by modelling the plant response using finite-length digital filters, the fixed plant model is never a perfect estimate and, therefore, a practical sound profiling algorithm must be robust to errors in the modelled plant response.

In addition to robustness against plant modelling errors, it is also desirable for sound profiling algorithms to achieve the desired spectrum with the minimum control effort. Minimum 
control effort can only be achieved when both the command signal and the disturbance signal are in phase [19]. Using a high level of control effort in order to obtain a target spectrum will unnecessarily overload the sound generation equipment, including the amplifiers and control actuators. Although the APC-FxLMS sound profiling algorithm is robust against plant errors, this comes at the expense of a potentially large control effort [19]. Conversely, under ideal operating conditions, the PSC-FxLMS algorithm uses the minimum control effort, but is highly sensitive to plant modelling errors. At present, therefore, there is no algorithm that provides both robustness to plant modelling errors and minimal control effort requirements for all sound profiling conditions. Therefore, in this paper, a modified PSC-FxLMS algorithm is proposed, which is both robust to plant modelling errors and achieves the target spectrum with minimum control effort. This improvement is achieved by removing the requirement of a secondary path model, and introducing an intelligent adaptive hysteresis switching scheme, which allows the phase of the disturbance signal to be estimated directly, even in the presence of variations in the frequency of the disturbance signal or in the signal-to-noise ratio (SNR).

The rest of this paper is organized as follows. A brief introduction to the existing active sound profiling schemes is presented in Section II and the proposed modified scheme is introduced in Section III. The effectiveness of the proposed sound profiling method is tested using simulations in Section $\mathrm{IV}$, and concluding remarks are provided in Section V.

\section{Active Sound Profiling Algorithms}

In a standard ANC system, an error microphone is used to detect the residual noise, and this signal is used to adapt the weights of the controller using, for example, the FxLMS algorithm. The error signal can be expressed as [16]

$$
e(n)=d(n)+\mathbf{g}^{T} \mathbf{u}(n),
$$

where $d(n)$ is the disturbance signal produced by the primary noise source, $\mathbf{g}$ is the impulse response vector between the control actuator and error sensor, and $\mathbf{u}(n)$ is the vector of current and previous control signal outputs. In the case of active sound profiling, however, instead of minimizing the error measured at the error microphone, $e(n)$, the system minimizes a pseudo-error, $e^{\prime}(n)$, given by

$$
e^{\prime}(n)=e(n)-c(n),
$$

where $c(n)$ is the command signal, whose amplitude is specified as the level desired at the error microphone. The control filter weights, $\boldsymbol{w}(n)$, in the sound profiling algorithm can then be updated according to the FxLMS algorithm with the error signal replaced by the pseudo-error to give

$$
\boldsymbol{w}(n+1)=\boldsymbol{w}(n)-\mu e^{\prime}(n) \hat{\boldsymbol{r}}(n),
$$

where $\hat{\boldsymbol{r}}(n)$ is the filtered reference signal vector and $\mu$ is the step size. As the control filter weights converge, the pseudoerror, $e^{\prime}(n)$, tends towards zero, which, according to (2) means that the error signal, $e(n)$, converges towards the command signal, $c(n)$ [19]. Therefore, the key difference between the various sound profiling systems lies in the definition of the command signal and, in particular, its phase. A brief review of two previously proposed sound profiling algorithms, namely, the PSC-FxLMS algorithm and the APC-FxLMS algorithm, is provided below before introducing the proposed modified PSC-FxLMS (MPSC-FxLMS) algorithm.

\section{A. Phase-Scheduled-Command FxLMS (PSC-FxLMS)}

In the PSC-FxLMS sound profiling scheme, the phase of the command signal is defined based on the phase of an estimate of the disturbance signal $\hat{d}(n)$ [19]. A block diagram of a sound profiling system implemented using the PSCFxLMS algorithm is shown in Fig. 1. The disturbance signal is estimated using an internal model of the plant response, $\hat{G}(z)$, such that the estimated disturbance signal can be expressed as

$$
\hat{d}(n)=e(n)-\hat{\mathbf{g}}^{T} \mathbf{u}(n)=d(n)+\mathbf{g}^{T} \mathbf{u}(n)-\hat{\mathbf{g}}^{T} \mathbf{u}(n)
$$

where $\hat{\mathbf{g}}^{T}$ is the impulse response vector of the internal plant model $\hat{G}(z)$. The estimated disturbance signal at the reference frequency can also be expressed using complex notation as

$$
\begin{aligned}
\hat{D} e^{j \phi_{\hat{d}}} & =E e^{j \phi_{e}}-U e^{j \phi_{u}} \hat{G} e^{j \phi_{\hat{g}}} \\
& =D e^{j \phi_{d}}+U e^{j \phi_{u}} G e^{j \phi_{g}}-U e^{j \phi_{u}} \hat{G} e^{j \phi_{\hat{g}}} \\
& =D e^{j \phi_{d}}+U e^{j \phi_{u}}\left[G e^{j \phi_{g}}-\hat{G} e^{j \phi_{\hat{g}}}\right]
\end{aligned}
$$

where $D$ and $\phi_{d}$ are the magnitude and phase of the disturbance signal, $U$ and $\phi_{u}$ are the magnitude and phase of the control signal, $E$ and $\phi_{e}$ are the magnitude and phase of the error signal, $G$ and $\phi_{g}$ are the magnitude and phase of the plant response $G(z)$, and the circumflex indicates an estimated or modeled value. From (4) and (5) it can be seen that the disturbance estimate, $\hat{d}(n)$, is dependent on both the magnitude, $\hat{G}$, and phase, $\phi_{\hat{g}}$, of the internal plant model $\hat{G}(z)$. In practice, the algorithm is somewhat sensitive to magnitude errors, but is particularly sensitive to phase errors in the internal plant model for high enhancement gains [19].

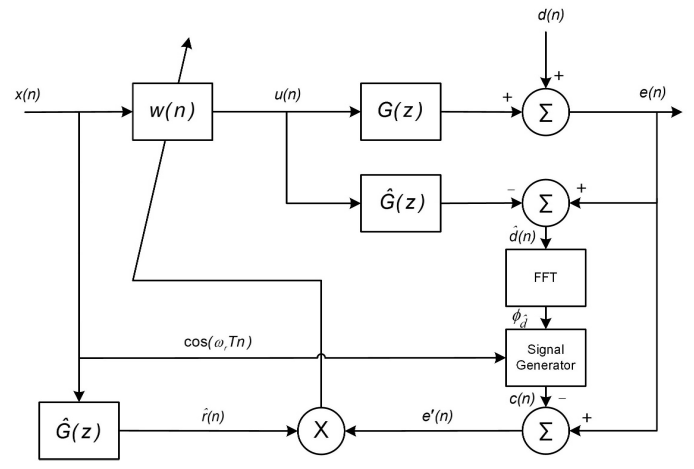

Fig. 1. Block diagram of the PSC-FxLMS algorithm.

\section{B. Automatic-Phase-Command FxLMS (APC-FxLMS)}

In order to improve the robustness of the PSC-FxLMS algorithm when the sound profiling algorithm is operating 
in high-enhancement mode, the APC-FxLMS algorithm was also proposed in [19]. The block diagram of the APC-FxLMS based sound profiling scheme is shown in Fig. 2. In the APC-FxLMS algorithm, the phase of the command signal is computed as

$$
\phi_{c}=\left\{\begin{aligned}
\phi_{\hat{d}} & \text { if } C \leq \hat{D} \\
\frac{2 \hat{D}}{C+\hat{D}} \phi_{\hat{d}} & \text { if } C>\hat{D}
\end{aligned}\right.
$$

where $C$ is the magnitude of the command signal. Although the APC-FxLMS algorithm provides improved robustness over the PSC-FxLMS algorithm, it does so at the expense of increased control effort due to the command signal phase being a biased estimate of the disturbance-signal phase $\phi_{\hat{d}}$ when $C>\hat{D}$, as per (6).

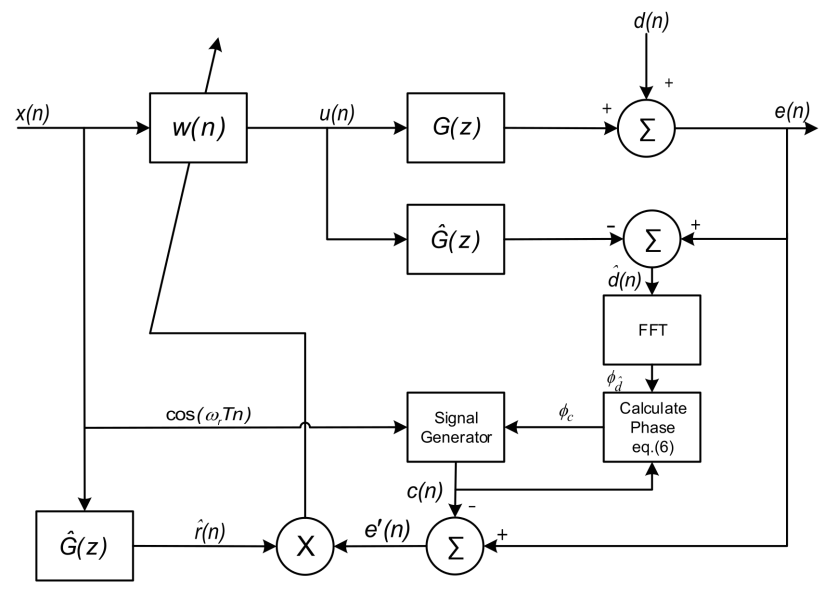

Fig. 2. Block diagram of the APC-FxLMS algorithm.

\section{PROPOSED MPSC-FxLMS AlgORITHM}

As discussed above, the PSC-FxLMS algorithm is highly sensitive to phase errors in the plant model and hence is not robust. Although the APC-FxLMS algorithm has been shown to improve the robustness, this comes at the expense of an increase in the required control effort. In practice, this means that high-power amplifiers and loudspeakers would be required, which would increase the cost and reduce the efficiency of the system. To overcome the limitations of the two algorithms discussed above, a sound profiling algorithm is proposed that is both robust to plant modelling errors and requires a minimal level of control effort. The reduced robustness of the PSC-FxLMS algorithm and the increased control effort necessary in the APC-FxLMS algorithm can be attributed to the errors in the estimation of the internal plant model, which in turn results in errors in estimating the phase of the disturbance signal [19].

Conceptually, accurate estimation of the phase of the disturbance signal could be achieved if the disturbance signal could be measured. However, this signal is not directly available, as the error microphone measures a superposition of the disturbance signal and the sound produced by the control loudspeaker. One method by which the disturbance signal could be measured is to switch off the control signal for a very short time period. During this period, the signal measured by the error microphone would be dominated by the disturbance signal, which could be used to estimate the phase of the disturbance signal. This task can be achieved by implementing an electronic switching scheme, which enables phase estimation whenever the magnitude of the error signal is more than a pre-defined command level. However, in a practical scenario, where the level of background noise may vary over time, this switching mechanism may lead to frequent switching even in cases where there is no variation in the disturbance signal and so this is not a practical approach.

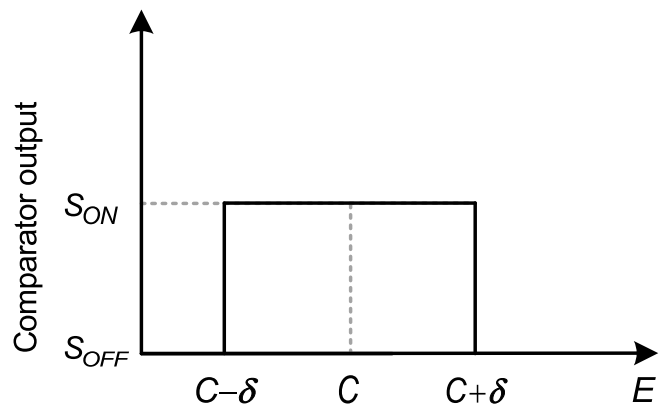

Fig. 3. Hysteresis switching characteristics.

The limitation of the above-mentioned switching scheme could be overcome by implementing a hysteresis-comparator switching mechanism. A hysteresis comparator has inputs $C$, $E$, and $\delta$, where $C$ and $E$ are the magnitudes of the command and error signals, respectively, and $2 \delta$ is the hysteresis window for the error signal [20]. The hysteresis window is computed as $\delta=\gamma C$ where, $\gamma$ is the tolerance factor and generally $\gamma \leq 0.05$, which equates to an error of less than or equal to $5 \%$ of the command signal magnitude. The hysteresis comparator avoids rapid switching in the presence of noise in the error signal or due to slight variations in the command or disturbance signals. The characteristics of the hysteresis switching scheme are shown in Fig. 3, where it can be observed that when the magnitude of the error signal is within a prescribed range, i.e., $C-\delta<E \leq C+\delta$, the controller is enabled and the error signal converges towards the command signal. However, when there is a variation in the disturbance signal (which means that $E$ is no longer in the prescribed range), the comparator output goes to zero, causing the switch to open. With the switch open, there is no control output and the control filter weights are held constant. During this period the disturbance signal phase is estimated directly, without the need for a plant model. 

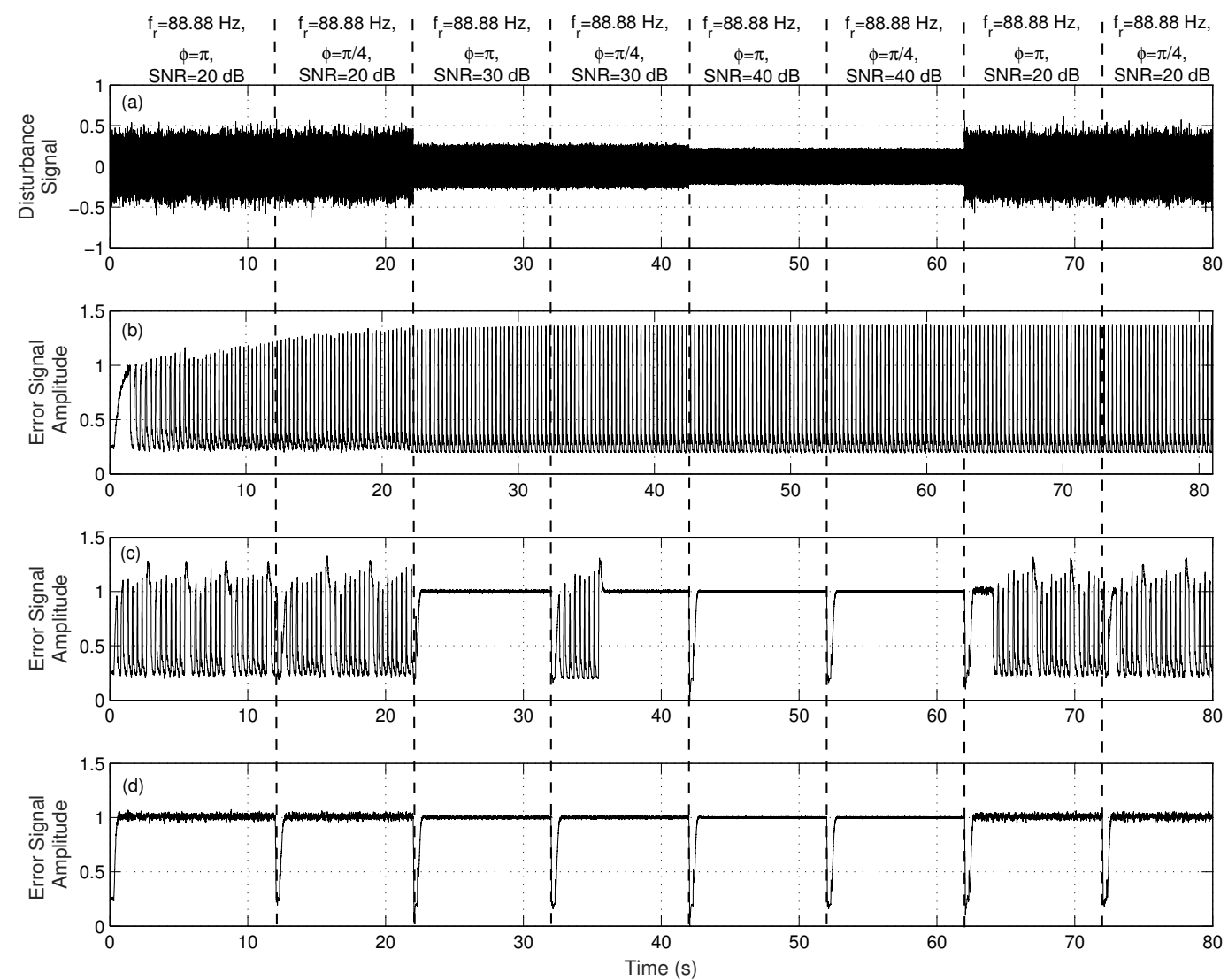

Fig. 4. Disturbance activity switch waveform. (a) Disturbance signal for different noise levels $20 \mathrm{~dB} \rightarrow 30 \mathrm{~dB} \rightarrow 40 \mathrm{~dB} \rightarrow 20 \mathrm{~dB}$. (b) Normal switching. (c) Hysteresis switching with constant threshold. (d) Hysteresis switching with dynamic threshold.

In scenarios where there is a variation in the SNR, the above-mentioned hysteresis switching mechanism may fail, i.e., when the switch is designed for a particular fixed level of SNR, $\gamma$, frequent switching will occur for lower SNR values. This issue can be observed in Fig. 4, where Fig. 4(a) shows the disturbance signal with different levels of SNR over different time intervals and Fig. 4(b) shows the switching pattern for a regular comparator. From this figure it can be seen that frequent switching occurs even in cases when there is no change in the SNR. The switching pattern for a hysteresis comparator designed for an SNR of $40 \mathrm{~dB}$ appears in Fig. 4(c), showing that in this case unwanted switching occurs when the SNR is lower than that for which the switch was designed.

In order to overcome this problem, an adaptive-hysteresis switching mechanism has been designed in which the threshold parameter, $\gamma$, is made a function of the estimated background noise level. The background noise $\eta(n)$ is estimated by computing the maximum magnitude of the residual error signal in a given window of $N$ samples, which is obtained as the difference between the error signal and the command signal. The threshold parameter is computed as $\gamma(n)=\alpha \eta(n)$, where $0<\alpha<0.5$ is a constant. Fig. 4(d) shows the switching pattern for the proposed adaptive-hysteresis switching scheme. The frequent switching issue of the conventional hysteresis switching scheme has been overcome, and the system can detect phase changes effectively.
In cases where the frequency of the disturbance signal changes, the adaptive hysteresis switching scheme will detect this variation through the difference between the magnitudes of the error and command signals. The switch will then turn OFF, and prevent the system from adapting the control signal to the new frequency. This is a limitation of the hysteresis-switchingbased sound profiling algorithm. In order to overcome this limitation, an attempt is made below to develop an intelligent adaptive hysteresis (IAH) switching scheme, which avoids turning the switch OFF when there is a difference between the frequencies of the command and error signals.

As discussed above, the controller must remain active even when there are changes in the frequency. As the signals considered in this work are of a single frequency, the switching scheme tracks the frequencies of interest for both the error and command signals. When the difference between those frequencies is greater than a small threshold $\left(\gamma_{f}\right)$, the controller must be kept active to allow the control signal to adapt to the new frequency; this can be achieved by keeping the switch ON. In this work, this requirement is implemented by using the frequency deviation measure between the command and error signal to enable or disable the adaptive-hysteresis switch. The sound profiling mechanism, which uses the proposed IAH switching scheme is updated using the MPSC-FxLMS algorithm. A block diagram of the proposed MPSC-FxLMS sound profiling method is shown in Fig. 5. In this approach, the 


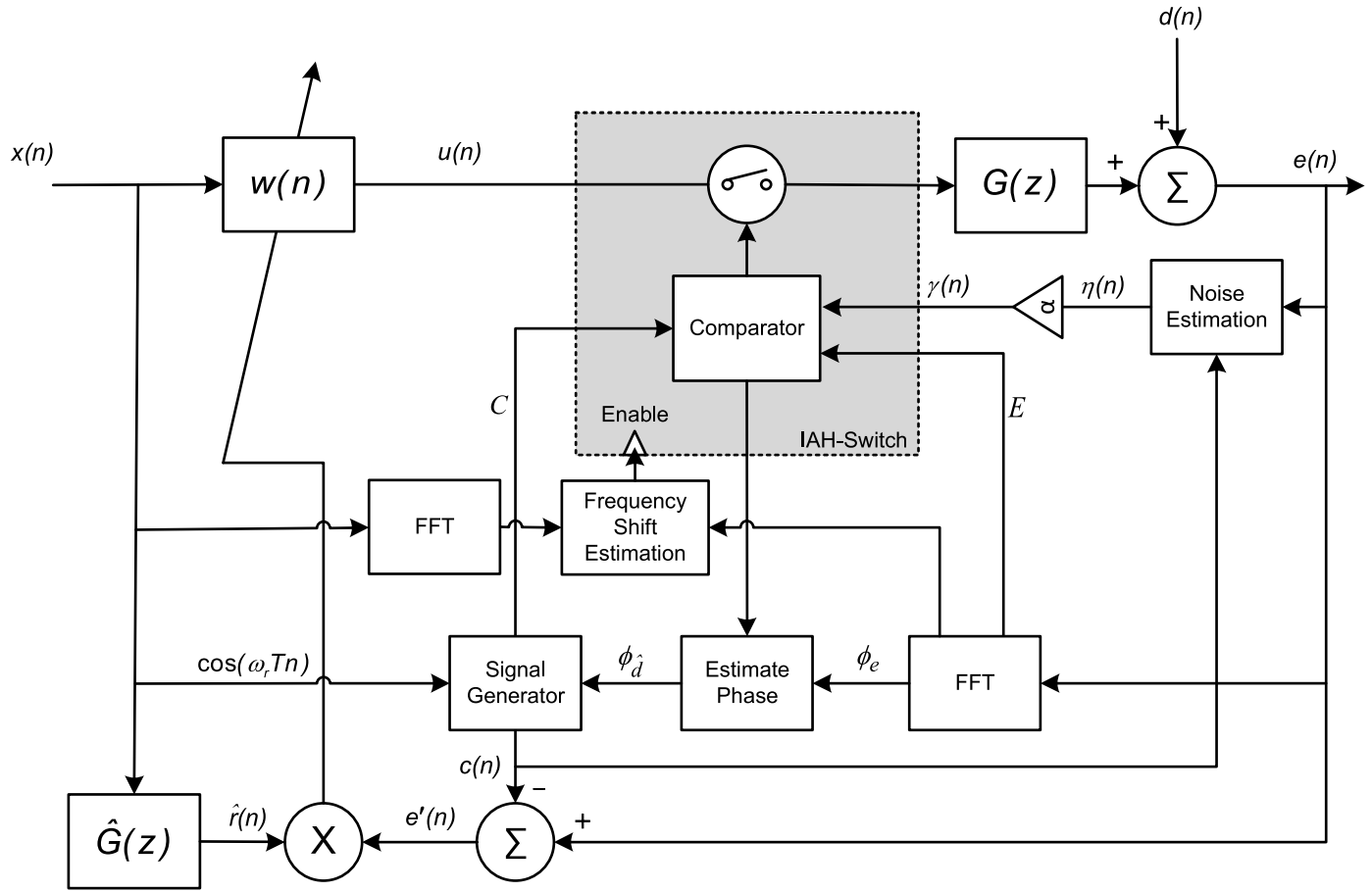

Fig. 5. Block diagram of the proposed MPSC-FxLMS algorithm.

internal plant model, $\hat{G}(z)$, used in APC-FxLMS based sound profiling has been removed and replaced with the proposed IAH switch. The switch turns the controller OFF, whenever a change in phase is detected and allows the phase of the disturbance signal to be measured directly.

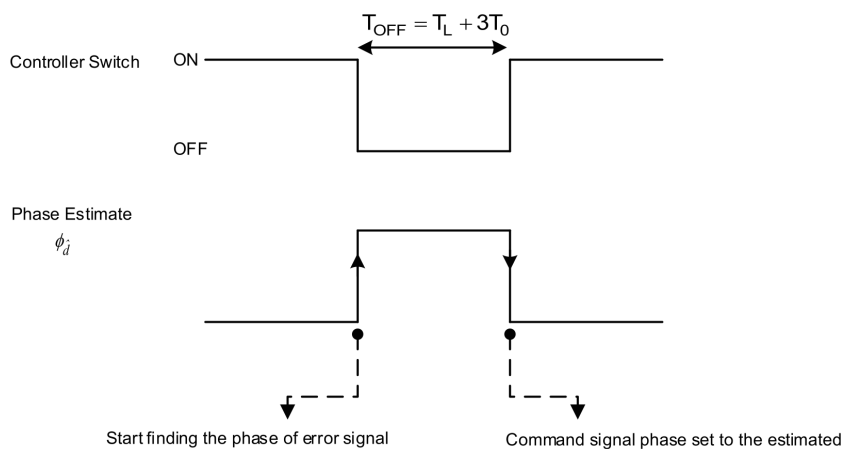

Fig. 6. Switching action of the proposed MPSC-FXLMS algorithm.

Fig. 6 summarizes the switching action and the disturbance signal phase estimation for the proposed algorithm. When the controller is OFF, the algorithm begins to calculate the phase of the error signal. The controller is switched OFF for $T_{\mathrm{OFF}}$ samples, the length of which will affect the behaviour of the controller and should be kept to a minimum in order to limit the period for which control is disabled. After this period, the controller is automatically switched $\mathrm{ON}$, and the command signal phase is set to the newly calculated value. A preliminary set of simulations determined that a switch off-time of $T_{\mathrm{OFF}}=$ $T_{\mathrm{L}}+3 T_{0}$ samples is sufficient to estimate the disturbance signal phase, where $T_{\mathrm{L}}$ is the number of samples in the plant
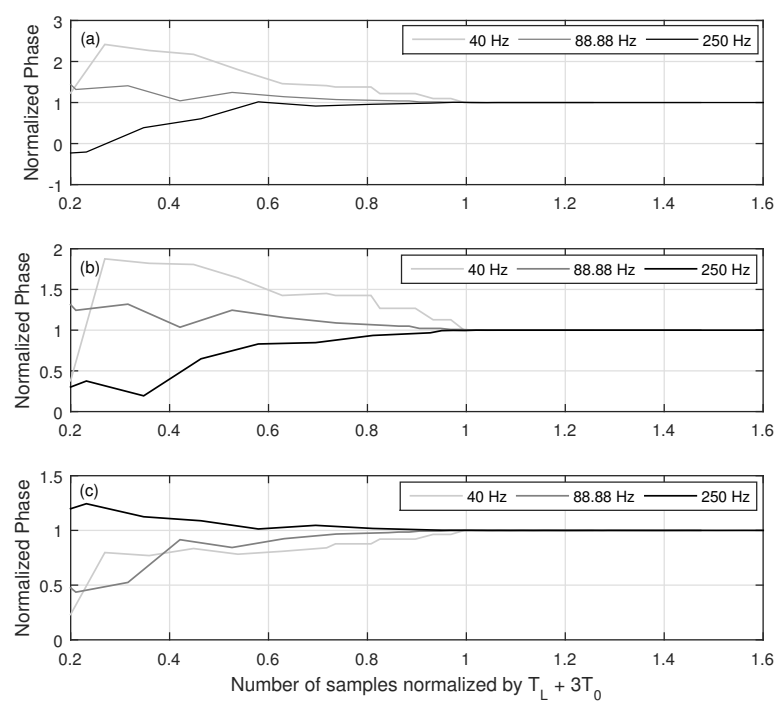

Fig. 7. Convergence characteristics of the disturbance signal phase estimate. (a) $\phi_{d}=0^{\circ}$. (b) $\phi_{d}=45^{\circ}$. (c) $\phi_{d}=135^{\circ}$.

impulse response, $\hat{\mathbf{g}}$, so that after $T_{\mathrm{L}}$ samples the error signal becomes equal to the disturbance signal $d(n)$, and $T_{0}$ is the number of samples in one period of the reference signal. The controller is switched ON automatically after $T_{\mathrm{OFF}}$ samples, and the command signal phase is set to the newly calculated value. Fig. 7 shows the results of a series of simulations to determine the necessary switch off-time, $T_{\mathrm{OFF}}$. Each sub-plot shows the convergence of the estimated phase, $\phi_{\hat{d}}$, normalised to the actual disturbance phase, $\phi_{d}$, such that a normalised 
TABLE I

SUMMARY OF THE PROPOSED MPSC-FXLMS ALGORITHM FOR ACTIVE SOUND PROFILING.

Step 1. Initialize the weights, $\boldsymbol{w}(n)$, of the adaptive controller with zeros

Step 2. Assign appropriate initial values for the number of samples $N$ for estimating the background noise $\eta(n)$, the step size $\mu$, frequency threshold parameter $\gamma_{f}$, the threshold parameter constant $\alpha$ and the command level $C$.

Step 3. Switch OFF (if the switch is in the ON state) the controller for $T_{\mathrm{OFF}}=T_{\mathrm{L}}+3 T_{0}$ samples, estimate the phase of the disturbance signal, $\phi_{\hat{d}}$, by computing the FFT of the error signal, $e(n)$, and embed the estimated disturbance signal phase in the command signal, i.e. $c(n)=C \cos \left(\omega_{r} T n+\phi_{\hat{d}}\right)$, where $\omega_{r}$ is the reference angular frequency and $T$ is the sampling period.

Step 4. Switch ON (if the switch is in the OFF state) the controller and update the weights of the adaptive filter using $\boldsymbol{w}(n+1)=\boldsymbol{w}(n)-\mu e^{\prime}(n) \hat{\boldsymbol{r}}(n)$, where $e^{\prime}(n)=e(n)-c(n)$ and $\hat{\boldsymbol{r}}(n)$ is the filtered reference signal vector.

Step 5. Estimate the background noise $\eta(n)$ for a given window of $N$ samples and then compute the threshold parameter as $\gamma(n)=\alpha \eta(n)$.

Step 6. Estimate the magnitude $E$ of the error signal and compute $\delta(n)=\gamma(n) C$.

Step 7. If $C-\delta(n)<E<C+\delta(n)$, the controller is kept ON and returns to Step 4.

Step 8. If $E$ is not in the range stated above and the estimate of the frequency deviation is less than the threshold, $\left|f_{c}-f_{e}\right| \leq \gamma_{f}$, then the controller is turned OFF and returned to Step 3 .

Step 9. If $E$ is not in the range stated in Step 7 and $\left|f_{c}-f_{e}\right|>\gamma_{f}$, then the controller is kept ON and returns to Step 4.

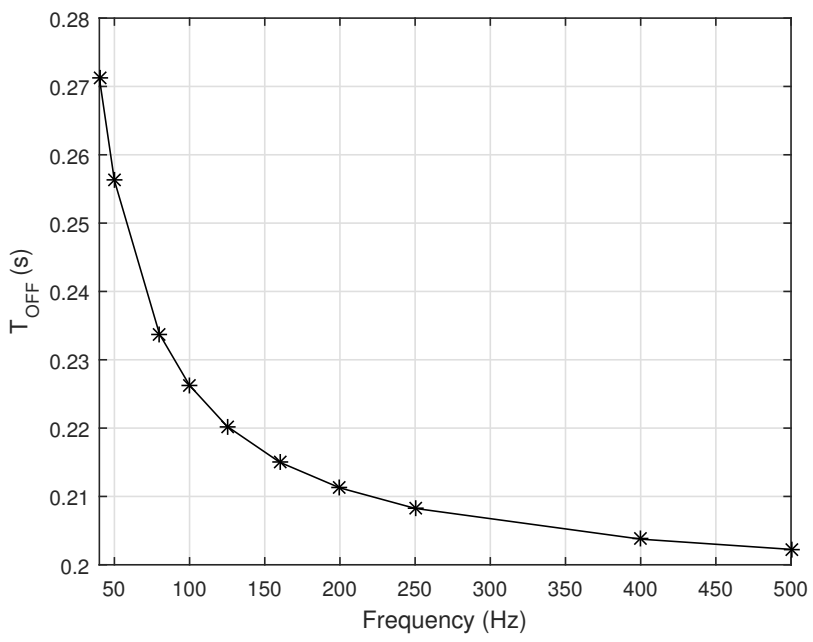

Fig. 8. Switch off-time, $T_{\mathrm{OFF}}$, as a function of reference signal frequency $F_{0}$.

phase of one indicates perfect estimation. The number of samples has also been normalised to $T_{\mathrm{OFF}}=T_{\mathrm{L}}+3 T_{0}$ and the results are shown for three different control frequencies. Each sub-plot in Fig. 7 shows the result of a different disturbance phase $\left(\phi_{d}=0^{\circ}, \phi_{d}=45^{\circ}\right.$, and $\left.\phi_{d}=135^{\circ}\right)$. From these results, it can be seen that the estimated phase converges to the disturbance phase within $T_{\mathrm{OFF}}=T_{\mathrm{L}}+3 T_{0}$ samples, irrespective of the actual disturbance phase or frequency. It should be highlighted, however, that $T_{\mathrm{OFF}}$ is a function of the reference signal frequency. Fig. 8 shows $T_{\mathrm{OFF}}$ for different reference signal frequencies, and from this plot, it can be seen that the switch off-time time reduces with increasing frequency, since $T_{0}$ is inversely proportional to the reference signal frequency. The switch off-time must be kept to a minimum in order to minimise the duration over which control is disabled. The steps followed in the proposed MPSC-FxLMS algorithm are summarised in Table I.

\section{Simulation StUdy}

The robustness and efficiency of the proposed sound profiling algorithm have been tested through a series of simulations.
Since the previously proposed sound profiling algorithms are particularly susceptible to plant errors when the enhancement gain is high [19], the following simulations initially compare the sound profiling algorithms operating in enhancement mode. The plant response used in the simulations was measured between a loudspeaker and a microphone, and both the impulse and frequency responses are shown in Fig. 9. To demonstrate the worst-case scenario, the command and disturbance signals have been set to be out of phase at the start of the simulation. The disturbance signal is a pure tone of $88.88 \mathrm{~Hz}$ with an SNR of $40 \mathrm{~dB}$, and the sampling frequency is $4 \mathrm{kHz}$. Other parameter values used in the simulations are: $D=0.2, C=1, N=3 T_{0}, \gamma_{f}=10, \alpha=0.2$, and $\mu=0.05$. The step size, $\mu$, has been chosen to maximize the convergence of each sound profiling algorithm.
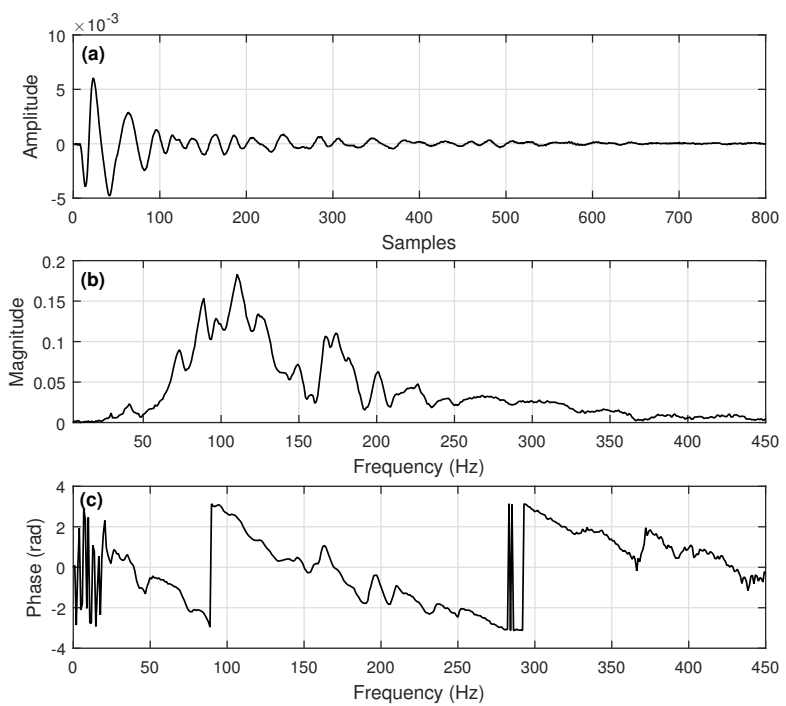

Fig. 9. Plant response used in simulation. (a) Impulse response $G(z)$. (b) Magnitude frequency response $G(z)$. (c) Phase frequency response $G(z)$.

Before comparing the performance of the proposed algorithm with other sound profiling algorithms, the effectiveness of the proposed method in the presence of changes in phase, frequency, and SNR is tested here. The tonal disturbance signal 


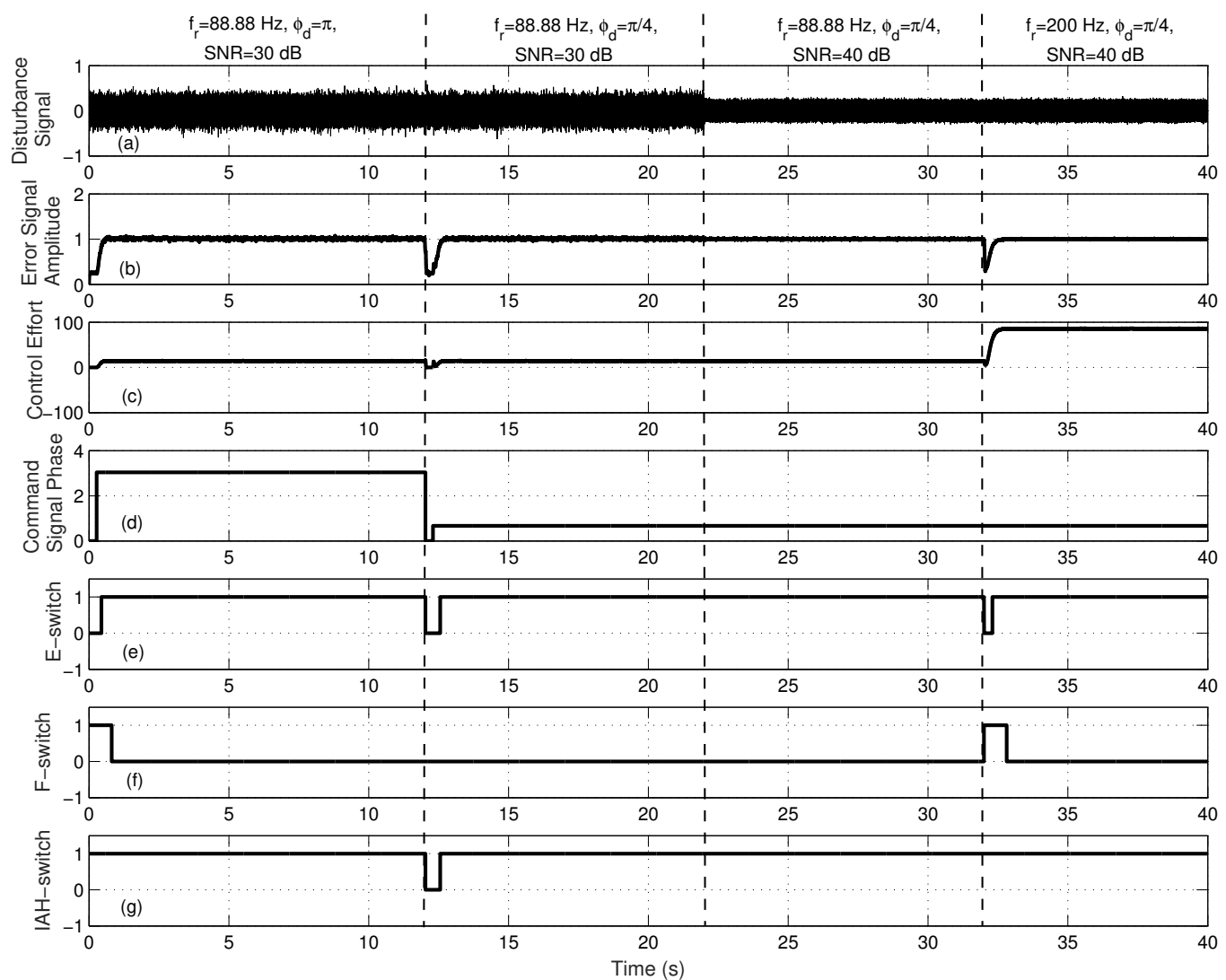

Fig. 10. Response of propsed method using IAH-switch, E-switch, and F-switch to change in phase, frequency, and SNR. (a) Disturbance signal. (b) Error signal variation for IAH-switch. (c) Control effort for MPSC-FxLMS algorithm for IAH-switch. (d) Command signal phase for IAH-switch. (e) Switching pattern for E-switch. (f) Switching pattern for F-switch. (g) Switching pattern for IAH-switch.

considered in this case is at $88.88 \mathrm{~Hz}$ between 0 and 31 seconds and at $200 \mathrm{~Hz}$ for the rest of the simulation. The phase of the disturbance signal varies from $\pi$ to $\pi / 4$ after $11 \mathrm{~s}$ and then remains constant until the end of the test. The SNR is kept as $30 \mathrm{~dB}$ until $21 \mathrm{~s}$ and is then changed to $40 \mathrm{~dB}$. The accuracy of the proposed switching method is compared in Fig. 10 to that of a switch that only detects changes in the magnitude of the error signal (E-switch) and a switch that can detect changes in frequency (F-switch). It can be observed from these results that the proposed IAH-switch is able to detect changes in the phase of the disturbance signal without introducing unnecessary switching due to changes in the frequency or SNR. It can also be observed that the control effort is relatively consistent for a disturbance signal of a particular frequency even when there are changes in the phase or SNR.

In order to compare the performance of proposed algorithm to the previous sound profiling strategies, the performance is first shown in Fig. 11 for the PSC-FxLMS, APCFxLMS, and MPSC-FxLMS algorithms with no plant error, i.e., $\hat{G}(z)=G(z)$. In Fig. 11(a), it can be seen that, in this case, convergence of the PSC-FxLMS algorithm is faster than for the other sound profiling algorithms, although all three algorithms do achieve the desired error signal level of 1. Fig. 11(b) shows that, for a perfect model, the PSC-FxLMS and
MPSC-FxLMS algorithms require a lower control effort than the APC-FxLMS algorithm, which is because the latter uses a command signal phase that is modified according to (6); the resulting phase difference can be seen in Fig. 11(c).

As previously discussed, the physical plant in the sound profiling system is likely to change over time and the algorithms must therefore remain stable in the presence of modelling errors. Fig. 12 compares the performance of the PSC-FxLMS, APC-FxLMS, and MPSC-FxLMS algorithms for the same conditions and parameter settings as above, but with a phase error of $8^{\circ}$, i.e. , $\hat{G}(z)=z^{-2} G(z)$. From Fig. 12(a), it can be seen that, due to this error in the plant model phase, the PSC-FxLMS algorithm starts to oscillate and never converges to the desired error signal level, whereas the other two algorithms do converge to 1. Fig. 12(b) shows the control effort required by the three sound profiling algorithms and it can be seen that the APC-FxLMS algorithm is able to achieve the desired level, but this comes at the expense of a higher control effort than the MPSC-FxLMS algorithm. The increase in control effort required by the APC-FxLMS algorithm and the oscillatory behaviour of the PSC-FxLMS algorithm are both due to a difference between the command signal phase and the disturbance signal phase, as shown in Fig. 12(c). From these results, it is evident that the proposed MPSC-FxLMS algorithm is robust to plant modelling errors and, only requires 
minimal control effort.
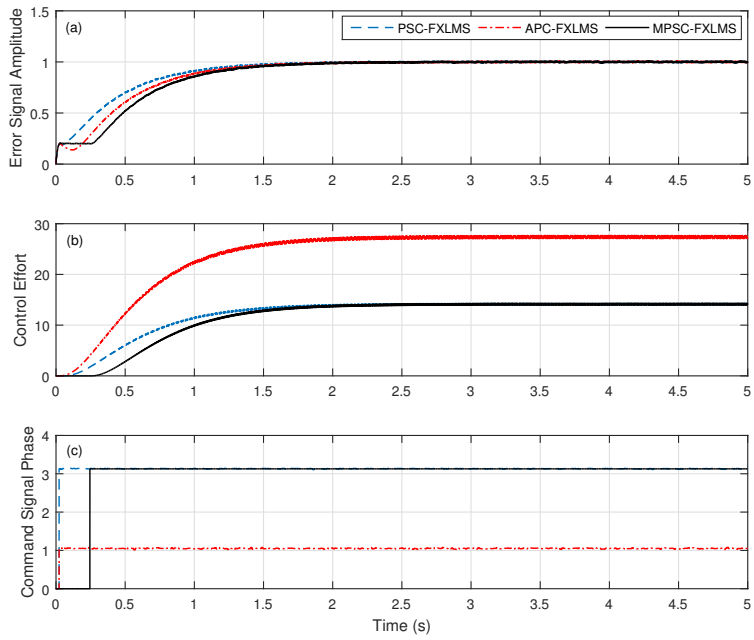

Fig. 11. Performance of the algorithms with no plant error $\hat{G}(z)=G(z)$. (a) Error signal amplitude. (b) Control effort. (c) Command signal phase.
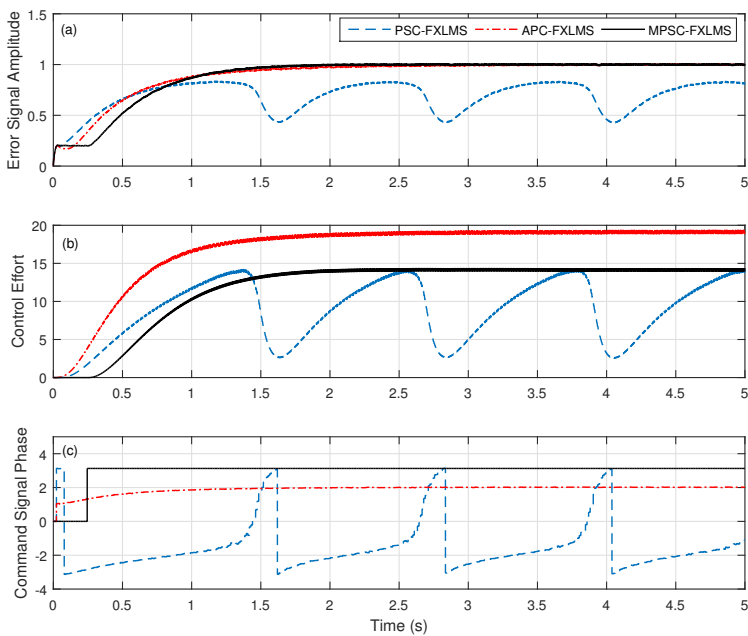

Fig. 12. Performance of the algorithms with phase error of 2 samples $\approx$ $8^{\circ}, \hat{G}(z)=z^{-2} G(z)$. (a) Error signal amplitude. (b) Control effort. (c) Command signal phase.

TABLE II

DIFFERENT MODES OF ACTIVE SOUND PROFILING

\begin{tabular}{c|c}
\hline Mode & Command level \\
\hline Cancellation & $C=0$ \\
Attenuation & $0<C<D$ \\
Neutralization & $C=D$ \\
Enhancement & $C>D$ \\
\hline
\end{tabular}

As shown in (5), that estimation of the disturbance signal phase is dependent on both the magnitude and phase of the plant estimate, $\hat{G}(z)$. Therefore, in order to also demonstrate the robustness and stability of the proposed algorithm to
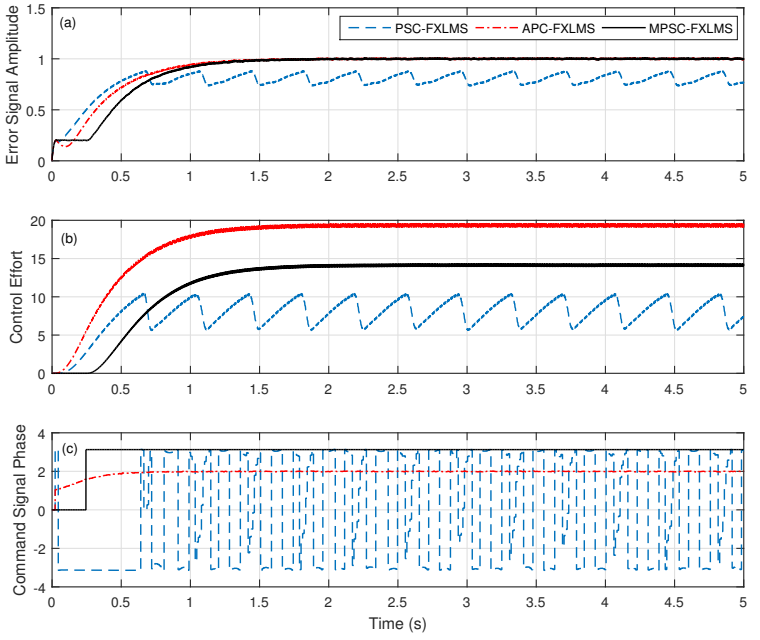

Fig. 13. Performance of the algorithms with magnitude error $\hat{G}(z)=$ $1.3 G(z)$. (a) Error signal amplitude. (b) Control effort. (c) Command signal phase.
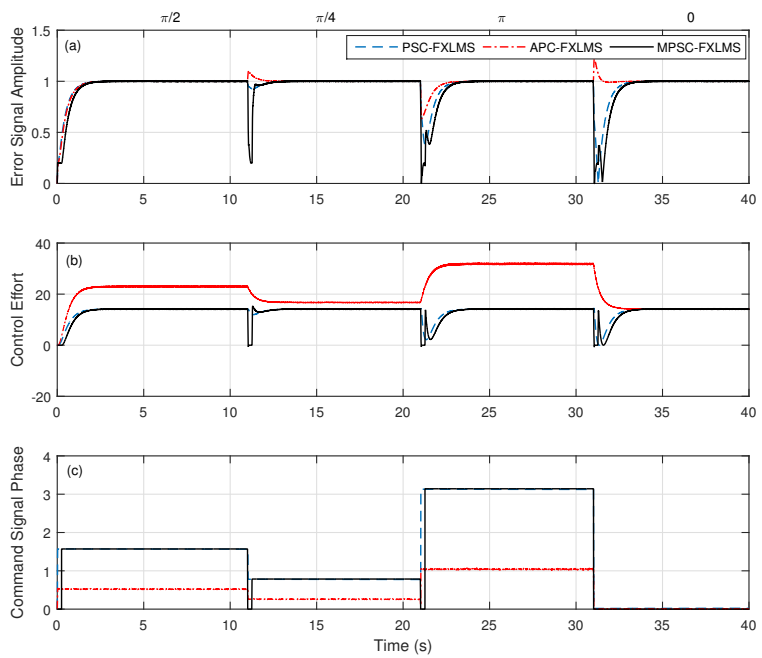

Fig. 14. Performance of the algorithms when tracking disturbance phase changes with no plant error $\hat{G}(z)=G(z)$. (a) Error signal amplitude. (b) Control effort. (c) Command signal phase.

magnitude errors in the plant model, an experiment was conducted with a magnitude error of $\hat{G}(z)=1.3 G(z)$. Fig. 13(a) shows that in the presence of magnitude errors in the plant estimate, the PSC-FxLMS algorithm never achieves the desired enhancement, whilst the APC-FxLMS algorithm reaches the desired level, but with a much larger control effort compared to the MPSC-FxLMS algorithm, as shown in Fig. 13(b). The reason for this increase in the control effort required by the APC-FxLMS algorithm is again due to the command signal phase calculation, and the difference between the command signal phase for the three algorithms can be seen in Fig. 13(c).

In order to demonstrate the tracking capability of the 

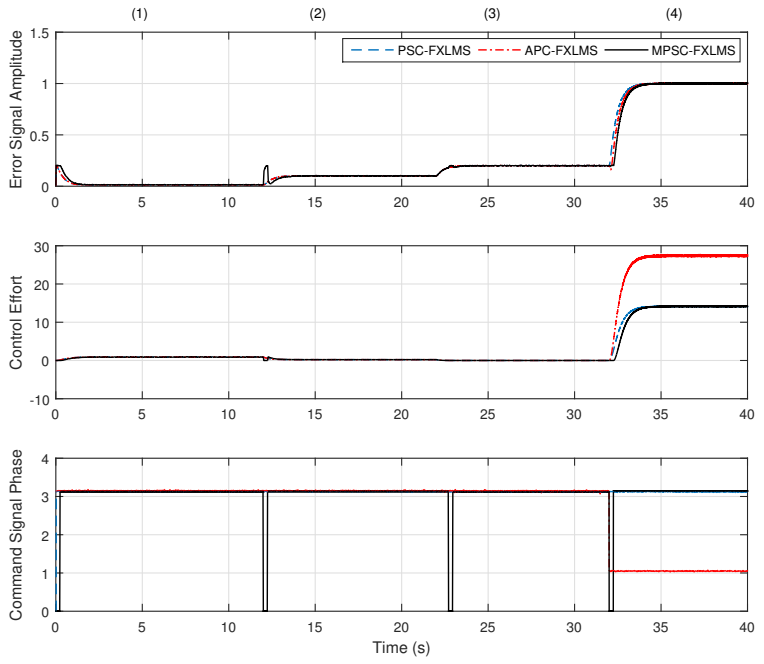

Fig. 15. Performance of the algorithms in different operating modes (Table.II) with no plant error $\hat{G}(z)=G(z)$ : (1) cancellation mode $(C=0)$, (2) attenuation mode $(C=0.1)$, (3) neutral mode $(C=0.2)$, and (4) enhancement mode $(C=1)$. (a) Error signal amplitude. (b) Control effort. (c) Command signal.

proposed algorithm, an experiment was conducted in which the phase of the disturbance signal changes from $\pi / 2 \rightarrow$ $\pi / 4 \rightarrow \pi \rightarrow 0$ after 10-s intervals. Fig. 14(a) shows the tracking capability of the proposed scheme along with that of the PSC-FxLMS and APC-FxLMS algorithms. It can be seen from Fig. 14(a) that the error signal in the proposed algorithm tends towards the disturbance level whenever there is a change in the phase of disturbance signal, i.e., when the controller is switched off. However, it should also be noted that the PSCFxLMS and APC-FxLMS algorithms also introduce transients in the error signal amplitude when there is a change in the phase. From Fig. 14(b), it may be noted that the proposed scheme and the PSC-FxLMS algorithm require a lower control effort compared to the APC-FxLMS algorithm. It can also be observed from Figs.14(b) and 14(c) that if the command and disturbance signals are not in phase, then a larger control effort is required to achieve the desired enhancement.

To demonstrate the performance of the proposed sound profiling scheme in all four control modes shown in Table.II [19], a simulation was conducted in which the amplitude of the command signal is changed after 10-s intervals to cycle through the control modes. It can be seen from Fig. 15(a) that for the proposed algorithm, the output error signal level goes to the disturbance level whenever the controller is switched off, as previously observed. From Figs .15(b) and 15(c) it can be seen that in the enhancement mode, the APC-FxLMS algorithm requires a larger control effort to achieve the desired level due to the phase adjustment governed by (6), whilst the PSC-FxLMS and proposed algorithms achieve the required performance with lower control effort. It should be noted that unlike in ANC algorithms, which attempt to drive the error signal to zero, in active sound profiling algorithms the error signal converges to the desired command level, as demon- strated in the presented results. The proposed MPSC-FxLMS algorithm has been shown to reach the desired command level with a minimal level of control effort.

\section{CONCLUSIONS}

A modified phase-scheduled-command FxLMS (MPSCFxLMS) algorithm has been proposed in this paper for active sound profiling. The proposed scheme has been shown to be robust against errors in both the magnitude and phase of the plant model, whilst using a minimal level of control effort. The proposed algorithm therefore improves the robustness compared to the previously proposed PSC-FxLMS algorithm, whilst avoiding high levels of control effort associated with the APC-FxLMS algorithm in the enhancement mode. These improvements in performance have been achieved by developing an intelligent adaptive hysteresis (IAH) switching scheme that allows the phase of the disturbance signal to be detected directly without the need for an additional internal plant model. The proposed algorithm, therefore, offers practical advantages over the previously proposed sound profiling strategies.

\section{REFERENCES}

[1] P. A. Nelson and S. J. Elliott, Active Control of Sound. Academic press, 1991.

[2] S. Elliott, I. Stothers, and P. Nelson, "A multiple error LMS algorithm and its application to the active control of sound and vibration," IEEE Trans. Acoust., Speech, Signal Process., vol. 35, no. 10, pp. 1423-1434, Oct. 1987.

[3] N. V. George and G. Panda, "Advances in active noise control: A survey, with emphasis on recent nonlinear techniques," Signal processing, vol. 93 , no. 2, pp. 363-377, 2013.

[4] J. Cheer and S. J. Elliott, "The design and performance of feedback controllers for the attenuation of road noise in vehicles," International Journal of Acoustics and Vibration, vol. 19, no. 3, pp. 155-164, 2014.

[5] _ "Multichannel control systems for the attenuation of interior road noise in vehicles," Mechanical Systems and Signal Processing, vol. 60, pp. 753-769, 2015.

[6] —-, "Active noise control of a diesel generator in a luxury yacht," Applied Acoustics, vol. 105, pp. 209-214, 2016.

[7] S. M. Kuo and D. R. Morgan, Active Noise Control Systems: Algorithms and DSP Implementations. New York: Wiley, 1996.

[8] L. P. de Oliveira, K. Janssens, P. Gajdatsy, H. Van der Auweraer, P. S. Varoto, P. Sas, and W. Desmet, "Active sound quality control of engine induced cavity noise," Mechanical systems and signal processing, vol. 23, no. 2, pp. 476-488, 2009.

[9] J. A. Mosquera-Sánchez, W. Desmet, and L. P. de Oliveira, "A multichannel amplitude and relative-phase controller for active sound quality control," Mechanical Systems and Signal Processing, vol. 88, pp. 145$165,2017$.

[10] S. Elliott, P. Nelson, I. Stothers, and C. Boucher, "In-flight experiments on the active control of propeller-induced cabin noise," J. Sound Vib., vol. 140, no. 2, pp. 219-238, 1990.

[11] A. Bullmore, P. Nelson, and S. Elliott, "Theoretical studies of the active control of propeller-induced cabin noise," J. Sound Vib., vol. 140, no. 2, pp. 191-217, 1990.

[12] M. Trinder and P. Nelson, "Active noise control in finite length ducts," J. Sound Vib., vol. 89, no. 1, pp. 95-105, 1983.

[13] T. M. Kostek and M. A. Franchek, "Hybrid noise control in ducts," $J$. Sound Vib., vol. 237, no. 1, pp. 81-100, 2000.

[14] Y. Kajikawa, W.-S. Gan, and S. M. Kuo, "Recent advances on active noise control: open issues and innovative applications," APSIPA Trans. Signal Inf. Process., vol. 1, p. E3, 2012.

[15] E. Zwicker and H. Fastl, Psychoacoustics: Facts and Models. Springer Science \& Business Media, 2013, vol. 22.

[16] S. J. Elliott, Signal Processing for Active Control. London: Academic Press, 2001.

[17] S. M. Kuo and M. J. Ji, "Development and analysis of an adaptive noise equalizer," IEEE Trans. Speech Audio Process., vol. 3, no. 3, pp. 217-222, May 1995. 
[18] S. M. Kuo, A. Gupta, and S. Mallu, "Development of adaptive algorithm for active sound quality control," J. Sound Vib., vol. 299, no. 1, pp. 1221, 2007.

[19] L. E. Rees and S. J. Elliott, "Adaptive algorithms for active soundprofiling," IEEE Trans. Audio, Speech, Language Process., vol. 14, no. 2, pp. 711-719, Mar. 2006.

[20] J. E. Varrientos and E. Sánchez-Sinencio, "A 4-D chaotic oscillator based on a differential hysteresis comparator," IEEE Trans. Circuits Syst. I, vol. 45, no. 1, pp. 3-10, Jan. 1998.

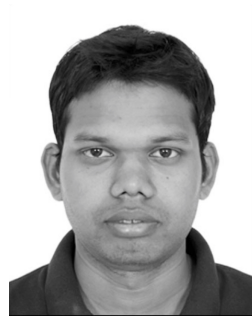

Vinal Patel received his Bachelor of Technology degree in Electronics and Telecommunication Engineering from the Chhattisgarh Swami Vivekananda Technical University, Bhilai, in 2010 and Master of Technology degree in Signal Processing from the Indian Institute of Technology, Guwahati, in 2013. Currently he is pursuing his Ph.D. degree in Electrical Engineering at Indian Institute of Technology Gandhinagar. His research interests include active noise control and signal processing.

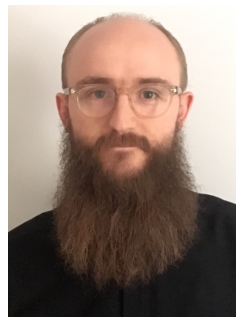

Jordan Cheer received the B.Mus. (Tonmeister) degree in music and sound recording from the Institute of Sound Recording, University of Surrey, Guildford, U.K., in 2008, and the M.Sc. and Ph.D. degrees in sound and vibration studies from the Institute of Sound and Vibration Research (ISVR), University of Southampton, Southampton, U.K., in 2009 and 2012, respectively.

From 2012 to 2014, he was a Research Fellow in Active Control at the ISVR. He was promoted to a Senior Research Fellow in 2015 and currently holds a New Frontiers Fellowship at the same institution. His research has focused on the active control of noise and vibration and array signal processing for audio reproduction.

Dr. Cheer is an Assistant Editor for the Journal of Sound and Vibration. He is a Chartered Engineer, a Member of the International Institute of Acoustics and Vibration, a Member of Institute of Mechanical Engineers and a Fellow of the Higher Education Academy. He received the P. E. Doak Award for academic performance in the taught part of the M.Sc., the E. J. Richards Prize for the best masters dissertation, and the Sir James Lighthill Award for the best student paper at the 19th International Congress on Sound and Vibration. $\mathrm{He}$ also served on the scientific committees for the 24th International Congress on Sound and Vibration and the 2016 Audio Engineering Society Conference on Sound Field Control.

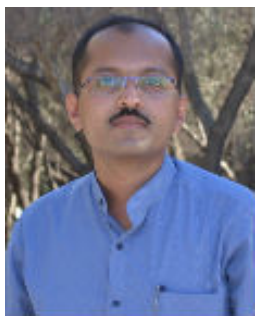

Nithin V. George (S'07 - M'09) received his Bachelor of Technology degree in Electronics and Communication Engineering from the University of Kerala, in 2007, Master of Technology degree in Telematics and Signal Processing from the National Institute of Technology, Rourkela, in 2009, and Ph.D. degree in Electrical Sciences from Indian Institute of Technology Bhubaneswar, in 2012. Currently he is working as an Assistant Professor in the discipline of Electrical Engineering at Indian Institute of Technology Gandhinagar. His research interests include active noise control, evolutionary computing and signal processing. 